\title{
MAGNETO-THERMO-MECHANICAL CREEP BEHAVIOR OF NANO-COMPOSITE ROTATING CYLINDER MADE OF POLYPROPYLENE REINFORCED BY MWCNTS
}

\author{
Abbas Loghman, Hossein Shayestemoghadam \\ Faculty of Mechanical Engineering, Department of Solid Mechanic, University of Kashan, Kashan, Iran \\ e-mail: aloghman@kashanu.ac.ir; h.shayestemoghadam@gmail.com
}

\begin{abstract}
History of strains, stresses and displacements of a rotating cylinder made of polypropylene reinforced by multi-walled carbon nanotubes (MWCNTs) subjected to magneto-thermo-mechanical loading is investigated using Burgers viscoelastic creep model. By making use of equations of equilibrium, stress-strain and strain-displacement, a constitutive differential equation containing creep strains is obtained which is solved semi analytically. It has been found that radial displacement, tangential strain and absolute values of radial strain are increasing with time at a decreasing rate so that they finally approach the steady state condition. Effective stresses are decreasing at the inner and increasing at the outer surface of the cylinder.
\end{abstract}

Keywords: nano-composite cylinder, creep analysis, MWCNTs, polypropylene, Burgers model

\section{Introduction}

Composite cylinders subjected to thermo-mechanical load are extensively used in aerospace, pressure vessels and petrochemical industries. Carbon nanotubes reinforced polymer matrix composites have shown high strength properties and are widely used in manufacturing of components exposed to high pressure environment. Even at room temperature, significant creep deformation is observed for polypropylene tubes. Therefore, creep analysis and creep life assessment of such components are very important. When such vessels are loaded, thermo-elastic stresses will be developed in the cylinder at zero time. However, because of creep evolution, stress redistribution occur during life of the component, which can affect its long-time performance.

The analysis of an internally pressurized, homogeneous, orthotropic rotating cylinder subjected to a steady state creep condition was investigated by Bhatnagar et al. (1984). In another work, they considered an orthotropic thick-walled cylinder under primary creep conditions (Bhatnagar et al., 1986). In their second work, the authors presented the analysis of an orthotropic, thick-walled cylinder undergoing creep due to combined action of internal and external pressures and rotary inertia. As a result, they found that the material which is strong in the radial direction may be beneficial for design of the cylinder as it gives lower values of the effective stress. Evaluation of creep compliances of unidirectional fiber-reinforced composites was done by Moal and Perreux (1994). In that paper, a method based on the model of Laws and McLaughlin was proposed for the determination of viscoelastic behavior of unidirectional fiber-reinforced composites. Moreover, the interface problem was taken into account by using anisotropic elastic coefficients for the reinforcement fiber. The suggested procedure allowed the viscoelasticity of the resin to be characterized. A variational method was developed by Ohno et al. (2002) for analyzing the matrix creep induced time-dependent change in fiber stress profiles in unidirectional composites. They verified the solutions on the basis of an energy balance equation and a finite 
difference computation. They also showed that the solution for the fiber pull-out model agreed well with an experiment on a single carbon fiber/acrylic model composite if the initial slip at fiber/matrix interface was taken into account. Singh and Ray (2002) modeled anisotropy and creep in an orthotropic aluminum-silicon carbide composite rotating disc. They observed that the anisotropy helped reduction of the tangential strain rate significantly, more near the inner radius. It was also found the strain rate distribution in the orthotropic disc was lower than that of isotropic disc following von Misses criterion. Creep deformations and stresses in thick-walled cylindrical vessels of functionally graded materials subjected to internal pressure were investigated by You et al. (2007). They examined how variations of material parameters along the radial direction affect the stresses in the vessels. Yang et al. (2006) carried out characterization of tensile creep resistance of polyamide 66 nano-composites. To develop their works, they presented both a viscoelastic creep model named Burgers model and an empirical method called Findley power law. They revealed that the simulation results from both models agreed quite well with the experimental data. Jia et al. (2011) studied the creep and recovery of polypropylene/multi-walled carbon nanotube composites. They showed that the creep strain reduces with a decrease in temperature and an increase in the content of carbon nanotubes. Magneto-thermo-elastic creep analysis of functionally graded cylinders was presented by Loghman et al. (2010). The paper describes time-dependent creep stress redistribution analysis of a thick-walled FGM cylinder subjected to a uniform magnetic field, temperature field and internal pressure. They calculated stress redistributions iteratively using magneto-thermo-elastic stresses as initial values for stress redistributions. The result indicated that the radial stress redistributions were not significant for different material properties, while major redistributions occurred for circumferential and effective stresses. A semi analytical solution of magneto-thermo-elastic stresses was suggested for functionally graded variable thickness rotating disks by Ghorbanpour Arani et al. (2010). In the paper, stresses and perturbation of magnetic field vector in FG rotating disks were determined using infinitesimal theory of magneto-thermo elasticity under plane stress conditions. It was found that imposing a magnetic field significantly decreases tensile circumferential stresses. Thus, the fatigue life of the disk would be significantly improved by applying the magnetic field. They suggested that the results of that investigation could be applied for optimum design of FG hollow rotating disks with variable thickness. Theory of plasticity for carbon nanotube reinforced composites was mentioned by Barai and Weng (2011). It was found that, with perfect interface contact, the decreasing of the CNT radius would improve the overall stiffness and plastic strength, but with an imperfect interface the size effect was reversed. Time-dependent thermo-elastic creep analysis of a rotating disk made of $\mathrm{Al}-\mathrm{SiC}$ composite using Mendelson's method of successive elastic solution was presented by Loghman et al. (2011). They found that the stresses, displacement, and creep strains were changing with time at a decreasing rate so that after almost 50 years the solution approached the steady-state condition.

The main objective of this paper is to obtain history of creep stresses and deformations of a nano-composite rotating cylinder made of polypropylene reinforced by MWCNTs using Burgers viscoelastic creep model under magneto-thermo-mechanical loadings.

\section{Geometry, loading condition and material properties}

\subsection{Geometry and loading condition}

A long rotating thick-walled nano-composite cylinder made of polypropylene reinforced by MWCNTs with inner radius $r_{i}$ and outer radius $r_{o}$ is considered (Fig. 1). The cylinder is subjected to a uniform magnetic field in the axial direction and a uniform temperature field. The following data for geometry and loading conditions are used in this paper: $r_{o} / r_{i}=2$, 
MWCNTs content $=4.5 \%, \nu=0.45, T=80^{\circ} \mathrm{C}, \omega=52.35 \mathrm{rad} / \mathrm{s}, \mu_{0}=4 \pi \cdot 10^{-7} \mathrm{H} / \mathrm{m}$, $H_{Z}=1 \cdot 10^{8} \mathrm{~A} / \mathrm{m}$.
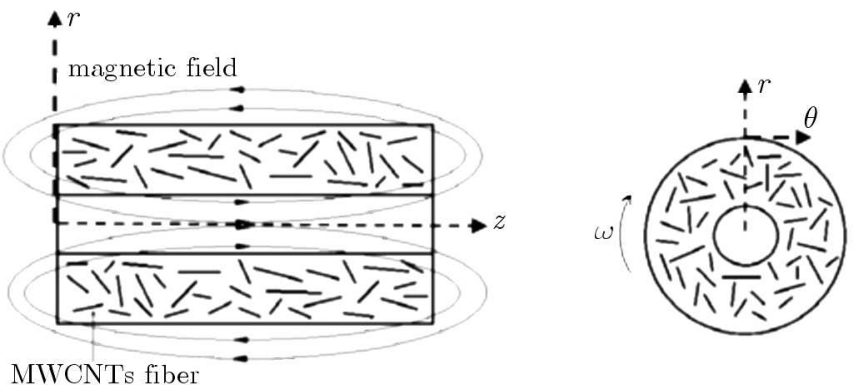

Fig. 1. Schematic of the rotating thick-walled composite cylinder subjected to uniform magnetic and thermal fields

\subsection{Material properties}

Young's modulus of the polymeric composite cylinder reinforced with different MWCNT contents is given in Table 1 based on experimental results reported by Jia et al. (2011).

Table 1. Young's modulus of propylene nano-composite with different MWCNT contents (Jia et al., 2011)

\begin{tabular}{|c|c|}
\hline MWCNTs content [vol.\%] & Young's modulus [GPa] \\
\hline \hline 0 & $1.83 \pm 0.11$ \\
\hline 0.3 & $2.10 \pm 0.11$ \\
\hline 0.6 & $2.12 \pm 0.03$ \\
\hline 2.8 & $2.33 \pm 0.14$ \\
\hline 4.5 & $2.42 \pm 0.13$ \\
\hline
\end{tabular}

\section{Burgers viscoelastic creep model}

Burgers four-element model can be used to predict the viscoelastic creep behavior of polymer based nano-composites. This model is shown in Fig. 2 with Maxwell and Kelvin rheological elements connected in series.

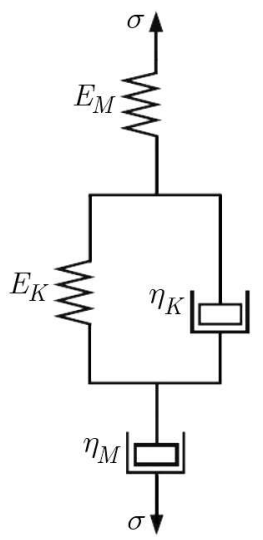

Fig. 2. Schematic diagram of Burgers four-element model (Yang et al., 2006) 
The creep constitutive model based on the Burgers law is written as follows (Yang et al., 2006)

$$
\varepsilon=\frac{\sigma_{0}}{E_{M}}+\frac{\sigma_{0}}{E_{K}}\left(1-\mathrm{e}^{\frac{-t}{\tau}}\right)+\frac{\sigma_{0}}{\eta_{M}} t \quad \tau=\frac{\eta_{K}}{E_{K}}
$$

in which $\sigma_{0}$ is the initially applied stress, $\tau$ is the retardation time taken to produce $63.2 \%$ or $\left(1-\mathrm{e}^{-1}\right)$ of the total deformation in the Kelvin element, $E_{M}$ and $\eta_{M}$ are the elastic modulus and viscosity of the Maxwell spring and dashpot model, $E_{k}$ and $\eta_{k}$ are the elastic modulus and the viscosity of the Kelvin spring and dashpot model. The Burgers model which includes the essential elements can be satisfactorily applied to describe behavior of viscoelastic materials practically. Differentiating Eq. (3.1) with respect to time gives the strain rate constitutive equation of the Burgers model as

$$
\dot{\varepsilon}=\frac{\sigma_{0}}{\eta_{M}}+\frac{\sigma_{0}}{\eta_{K}} \mathrm{e}^{\frac{-t}{\tau}}
$$

The material parameters, $\eta_{m}, E_{K}$ and $\eta_{k}$ are simulated from the experimental data (Jia et al., 2011).

Table 2. The simulated parameters of the Burger model with different MWCNT contents for long term prediction (Jia et al., 2011)

\begin{tabular}{|c|c|c|c|c|}
\hline MWCNTs [vol.\%] & $E_{k}[\mathrm{MPa}]$ & $\eta_{k}[\mathrm{MPa}]$ & $\eta_{m}[\mathrm{~s}]$ & $\tau[\mathrm{s}]$ \\
\hline \hline 0 & 5.7 & $9.00 \mathrm{E}+07$ & $1.50 \mathrm{E}+10$ & $1.49 \mathrm{E}+07$ \\
\hline 0.3 & 8.5 & $1.00 \mathrm{E}+08$ & $2.10 \mathrm{E}+10$ & $1.53 \mathrm{E}+07$ \\
\hline 0.6 & 9.2 & $1.50 \mathrm{E}+08$ & $2.60 \mathrm{E}+10$ & $1.63 \mathrm{E}+07$ \\
\hline 2.8 & 9.6 & $1.70 \mathrm{E}+08$ & $2.70 \mathrm{E}+10$ & $1.77 \mathrm{E}+07$ \\
\hline 4.5 & 10.4 & $2.00 \mathrm{E}+08$ & $2.80 \mathrm{E}+10$ & $1.92 \mathrm{E}+07$ \\
\hline
\end{tabular}

\section{Theoretical analysis}

The strain-displacement relationship for a long cylinder under an axisymmetric loading condition is written as

$$
\varepsilon_{r}=\frac{\partial u_{r}}{\partial r} \quad \varepsilon_{\theta}=\frac{u_{r}}{r}
$$

where $\varepsilon_{r}$ and $\varepsilon_{\theta}$ are the radial and circumferential total strains, and $u_{r}$ is the radial displacement.

Considering the total strains to be the sum of elastic, thermal and creep strains, the stress-strain relations may be written as follows

$$
\begin{aligned}
& \sigma_{r}=C_{11} \varepsilon_{r}+C_{12} \varepsilon_{\theta}-\lambda_{r} T_{r}-\left(C_{11} \varepsilon_{r}^{c}+C_{12} \varepsilon_{\theta}^{c}\right) \\
& \sigma_{\theta}=C_{21} \varepsilon_{r}+C_{22} \varepsilon_{\theta}-\lambda_{\theta} T_{r}-\left(C_{21} \varepsilon_{r}^{c}+C_{22} \varepsilon_{\theta}^{c}\right)
\end{aligned}
$$

where $\sigma_{r}$ and $\sigma_{\theta}$ are the radial and circumferential stresses, $\varepsilon_{r}^{c}$ and $\varepsilon_{\theta}^{c}$ are the radial and circumferential creep strains, $T_{r}$ is the temperature field. and the other coefficients are defined as

$$
\begin{array}{lll}
C_{11}=\frac{E(1-\nu)}{(1+\nu)(1-2 \nu)} & C_{12}=\frac{E v}{(1+\nu)(1-2 \nu)} & C_{21}=C_{12} \\
C_{22}=C_{11} & \lambda_{r}=\frac{E \alpha}{1-2 \nu} & \lambda_{\theta}=\frac{E}{1+\nu}
\end{array}
$$


in which $E, \nu$ and $\alpha$ are Young's modulus, Poison's ratio and the coefficient of thermal expansion of nano-composite, respectively.

The equilibrium equation of a thick-walled composite hollow cylinder subjected to a uniform magnetic field is written as(Loghman et al., 2010)

$$
\frac{\partial \sigma_{r}}{\partial r}+\frac{\sigma_{r}-\sigma_{\theta}}{r}+f_{r}+\rho r \omega^{2}=0
$$

in which $\rho r \omega^{2}$ is the centrifugal body force per unit volume, and $f_{r}$ is the Lorentz force written as

$$
f_{r}=\mu_{(r)} H_{z}^{2} \frac{\partial}{\partial r}\left(\frac{\partial u_{r}}{\partial r}+\frac{u_{r}}{r}\right)
$$

where $\mu_{(r)}$ is the magnetic permeability and $H_{z}$ is the magnetic field intensity in the axial direction. Substituting the strains from Eqs. (4.1) into Eqs. (4.2), and then substituting the radial and circumferential stresses into equilibrium Eq. (4.4) and substituting $f_{r}$ from Eq. (4.5), the following constitutive differential equation for displacement is obtained

$$
\begin{aligned}
& \left(C_{11}+\mu_{(r)} H_{z}^{2}\right) \frac{\partial^{2} u_{r}}{\partial r^{2}}+\left(\frac{C_{11}}{r}+\frac{\mu_{(r)}}{r} H_{z}^{2}\right) \frac{\partial u_{r}}{\partial r}+\left(\frac{C_{22}}{r^{2}}-\frac{H_{z}^{2}}{r^{2}} \mu_{(r)}\right) u_{r} \\
& =\frac{\lambda_{r}-\lambda_{\theta}}{r} T_{r}+\lambda_{r} \frac{\partial T_{r}}{\partial r}+\frac{\varepsilon_{r}^{c}\left(C_{11}-C_{21}\right)}{r}+\frac{\varepsilon_{\theta}^{c}\left(C_{12}-C_{22}\right)}{r}+\frac{\partial}{\partial r}\left(C_{11} \varepsilon_{r}^{c}+C_{22} \varepsilon_{\theta}^{c}\right)-\rho r \omega^{2}
\end{aligned}
$$

The above differential equation is summarized as

$$
D_{11} \frac{\partial^{2} u_{r}}{\partial r^{2}}+D_{12} \frac{\partial u_{r}}{\partial r}+D_{13} u_{r}+D_{14}=0
$$

in which

$$
\begin{aligned}
& D_{11}=C_{11}+\mu_{(r)} H_{z}^{2} \quad D_{12}=\frac{C_{11}}{r}+\frac{\mu_{(r)}}{r} H_{z}^{2} \quad D_{13}=\frac{C_{22}}{r^{2}}-\frac{H_{z}^{2}}{r^{2}} \mu_{(r)} \\
& D_{14}=\frac{\lambda_{r}-\lambda_{\theta}}{r} T_{r}+\lambda_{r} \frac{\partial T_{r}}{\partial r}+\frac{\varepsilon_{r}^{c}\left(C_{11}-C_{21}\right)}{r}+\frac{\varepsilon_{\theta}^{c}\left(C_{12}-C_{22}\right)}{r}+\frac{\partial}{\partial r}\left(C_{11} \varepsilon_{r}^{c}+C_{22} \varepsilon_{\theta}^{c}\right)-\rho r \omega^{2}
\end{aligned}
$$

$D_{14}$ contains creep strains which are time, temperature and stress dependent.

If we ignore the time-dependent creep strains in the coefficient $D_{14}$, then differential Eq. (4.7) becomes Navier's equation, a non-homogenous second-order ordinary differential equation with variable coefficients the solution to which can be found from magneto-thermo-elastic analysis. This analysis is done by making use of the division method (Hosseini Kordkheili and Naghdabai, 2007). In this method, the cylinder thickness is divided into a finite number of divisions. Then Navier's equation for $k$-th division yields the following differential equation with constant coefficients

$$
\left(D_{11}^{(k)} \frac{\partial^{2}}{\partial r^{2}}+D_{12}^{(k)} \frac{\partial}{\partial r}+D_{13}^{(k)}\right) u_{r}^{k}+D_{14}^{k}=0
$$

The coefficients of Eq. (4.9) are evaluated in each division in terms of constants and the radius of kth division. The exact solution to Eq. (4.9) can be written in the form of

$$
u_{r}^{(k)}=X_{1}^{(k)} \exp \left(\eta_{1}^{(k)} r^{(k)}\right)+X_{2}^{(k)} \exp \left(\eta_{2}^{(k)} r^{(k)}\right)-\frac{D_{14}^{(k)}}{D_{13}^{(k)}}
$$

where

$$
\eta_{1}^{(k)}, \eta_{2}^{(k)}=\frac{D_{12}^{(k)} \pm \sqrt{\left(D_{12}^{(k)}\right)^{2}-4 D_{13}^{(k)} D_{11}^{(k)}}}{2 D_{11}^{(k)}}
$$


It is noted that this solution to Eq. (4.9) is valid in the following sub-domain

$$
r^{(k)}-\frac{t^{(k)}}{2} \leqslant r \leqslant r^{(k)}+\frac{t^{(k)}}{2}
$$

where $t^{(k)}$ is the thickness of kth division and $X_{1}^{(k)}, X_{2}^{(k)}$ are unknown constants for $k$-th division. The unknowns $X_{1}^{(k)}$ and $X_{2}^{(k)}$ are determined by applying the necessary boundary conditions between two adjacent sub-domains. For this purpose, the continuity of the radial displacement $u$ as well as the radial stress $\sigma_{r}$ is imposed at the interfaces of the adjacent sub-domains. These continuity conditions at the interfaces are written as

$$
\begin{aligned}
& \left.u_{r}^{(k)}\right|_{r=r^{(k)}}+\frac{t^{(k)}}{2}=\left.u_{r}^{(k+1)}\right|_{r=r^{(k+1)}}-\frac{t^{(k+1)}}{2} \\
& \left.\sigma_{r}^{(k)}\right|_{r=r^{(k)}}+\frac{t^{(k)}}{2}=\left.\sigma_{r}^{(k+1)}\right|_{r=r^{(k+1)}}-\frac{t^{(k+1)}}{2}
\end{aligned}
$$

and the global boundary conditions are

$$
\sigma_{r}=0 \quad \text { at } \quad r=r_{i} \quad \sigma_{r}=0 \quad \text { at } \quad r=r_{o}
$$

Continuity conditions Eqs. (4.13) together with global boundary conditions Eqs. (4.14) yield a set of linear algebraic equations in terms of $X_{1}^{(k)}$ and $X_{2}^{(k)}$. Solving the resultant linear algebraic equations for $X_{1}^{(k)}$ and $X_{2}^{(k)}$, the unknown coefficients of Eq. (4.10) are calculated. Then, the displacement component ur and the stresses are determined in each radial sub-domain. Accuracy of the results will be improved by increasing number of divisions.

\section{Time-dependent creep analysis}

For time-dependent creep analysis, the creep strains in the coefficient $D_{14}$ must be considered. The creep strains are time, temperature, and stress dependent. Creep strain increments are related to the current stresses and the material uni-axial creep behavior by Prandtl-Reuss relations. For problems of a rotating thick-walled composite cylinder with axial symmetry, these relations (Loghman et al., 2010) are written as follows

$$
\begin{array}{ll}
\dot{\varepsilon}_{r}^{c} & =\frac{\dot{\varepsilon}_{c}}{2 \sigma_{e}}\left[2 \sigma_{r}-\left(\sigma_{\theta}+\sigma_{z}\right)\right] \quad \dot{\varepsilon}_{\theta}^{c}=\frac{\dot{\varepsilon}_{c}}{2 \sigma_{e}}\left[2 \sigma_{\theta}-\left(\sigma_{r}+\sigma_{z}\right)\right] \\
\dot{\varepsilon}_{z}^{c} & =\frac{\dot{\varepsilon}_{c}}{2 \sigma_{e}}\left[2 \sigma_{z}-\left(\sigma_{r}+\sigma_{\theta}\right)\right]
\end{array}
$$

where $\dot{\varepsilon}_{r}^{c}, \dot{\varepsilon}_{\theta}^{c}$ and $\dot{\varepsilon}_{z}^{c}$ are the radial, circumferential, and axial creep strain rates, $\dot{\varepsilon}_{c}$ and $\sigma_{c}$ are the equivalent creep strain rate and equivalent stress, respectively. These equivalent or effective variables are defined as follows

$$
\begin{array}{ll}
\dot{\varepsilon}_{c} & =\frac{2}{\sqrt{3}} \sqrt{\left(\dot{\varepsilon}_{r}^{c}\right)^{2}+\left(\dot{\varepsilon}_{\theta}^{c}\right)^{2}+\left(\dot{\varepsilon}_{z}^{c}\right)^{2}} \quad \sigma_{e}=\sqrt{\frac{1}{2}\left[\left(\sigma_{r}-\sigma_{\theta}\right)^{2}+\left(\sigma_{r}-\sigma_{z}\right)^{2}+\left(\sigma_{\theta}-\sigma_{z}\right)^{2}\right]} \\
\dot{\varepsilon}_{z}^{c} & =0 \rightarrow \sigma_{z}=\frac{1}{2}\left(\sigma_{r}+\sigma_{\theta}\right)
\end{array}
$$

The material creep constitutive model can be rewritten in terms of the equivalent creep strain rateand equivalent stress as

$$
\dot{\varepsilon}_{c}=\frac{\sigma_{0}}{\eta_{M}}+\frac{\sigma_{0}}{\eta_{K}} \mathrm{e}^{\frac{-t}{\tau}}
$$


Equations (5.1), (5.2), and (5.3) together with differential Eq. (4.9) are used in a numerical procedure based on Mendelson's method of successive elastic solution (Mendelson, 1968) to obtain history of stresses and deformations during creep process. The numerical procedure is explained by Loghman et al. (2011).

\section{Numerical results and discussion}

The results presented in this study are based on the data presented in Section 2 for geometry, material properties and loading conditions. The elastic properties are dependent on the volume percent of MWCNTs content and are given in Table 1. In this research, a $4.5 \%$ volume content of MWCNTs is considered.

The history of radial displacement, radial and circumferential stresses, effective stress, radial and circumferential strains, radial and circumferential creep strains are plotted with and without the effect of magnetic field in Figs. 3 to 10.

Figures $3 \mathrm{a}$ and $3 \mathrm{~b}$ show the radial displacement histories. Generally, the radial displacements are increasing with time at a decreasing rate during life of the cylinder so that finally approach the steady state condition. However, in the presence of a magnetic field, the radial displacements are lower in magnitude.
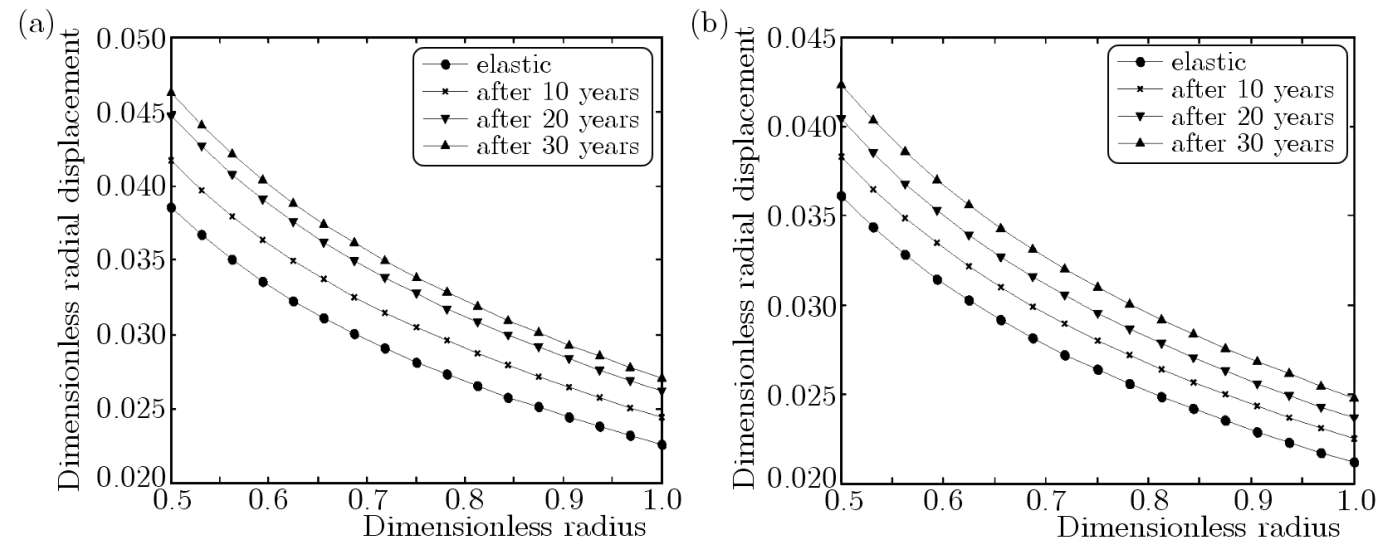

Fig. 3. Radial displacement of the nano-composite cylinder (a) without and (b) with the effect of magnetic field $(H=1 \mathrm{E}+8)$
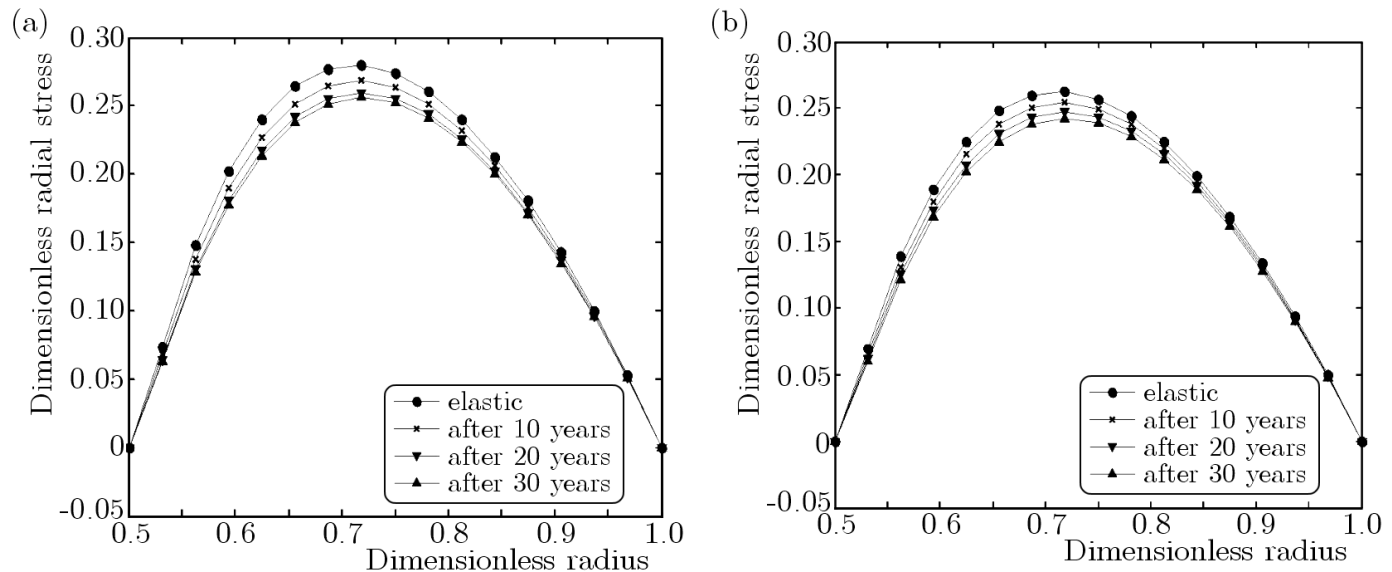

Fig. 4. Radial stress of the nano-composite cylinder (a) without and (b) with the effect of magnetic field $(H=1 \mathrm{E}+8)$ 
Histories of the radial stress are illustrated in Figs. 4a and 4b in which the boundary conditions at the inner and outer surfaces of the cylinder are satisfied. The radial stresses are decreasing with time during life of the cylinder. The radial stresses with the effect of magnetic field are of lower magnitudes.

Histories 9f the circumferential stress are illustrated in Figs. 5a and 5b. The circumferential stresses are decreasing at the inner surface of the cylinder and are increasing at the outer surface so that the reference point can be identified where the circumferential stress is not changing with time.
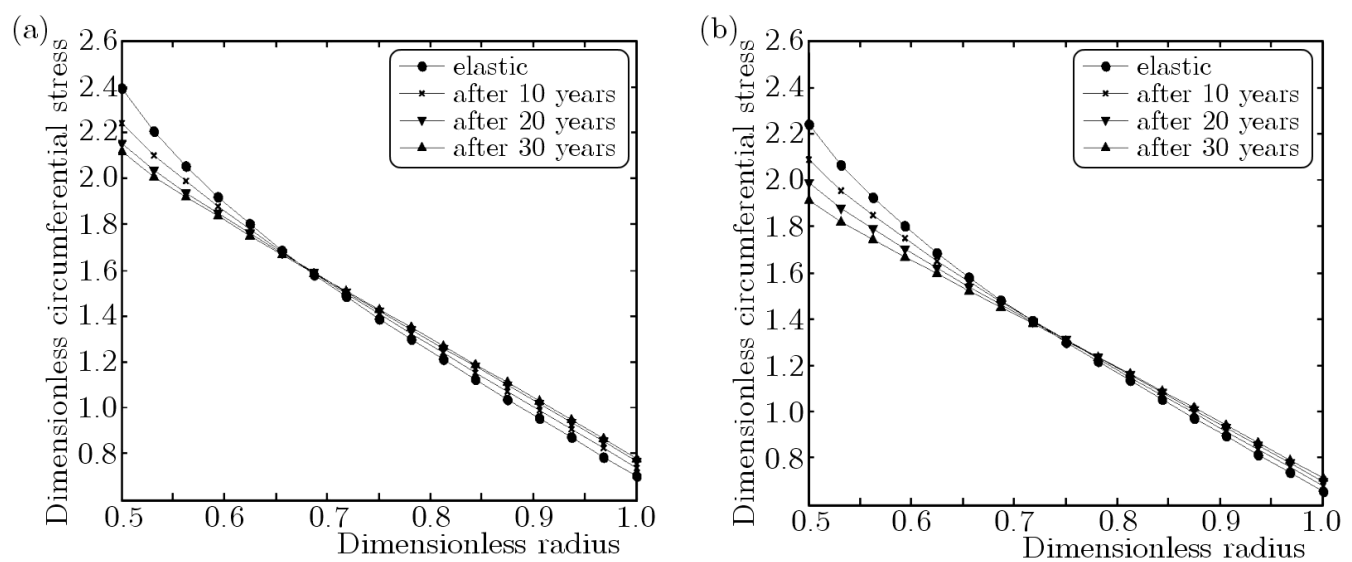

Fig. 5. Circumferential stress of the nano-composite cylinder (a) without and (b) with the effect of magnetic field $(H=1 \mathrm{E}+8)$

Histories of the effective stress are demonstrated in Figs. 6a and 6b. The effective stresses are very similar to circumferential stresses. This is because the circumferential stresses are almost ten times greater than the radial stresses and therefore are dominant.
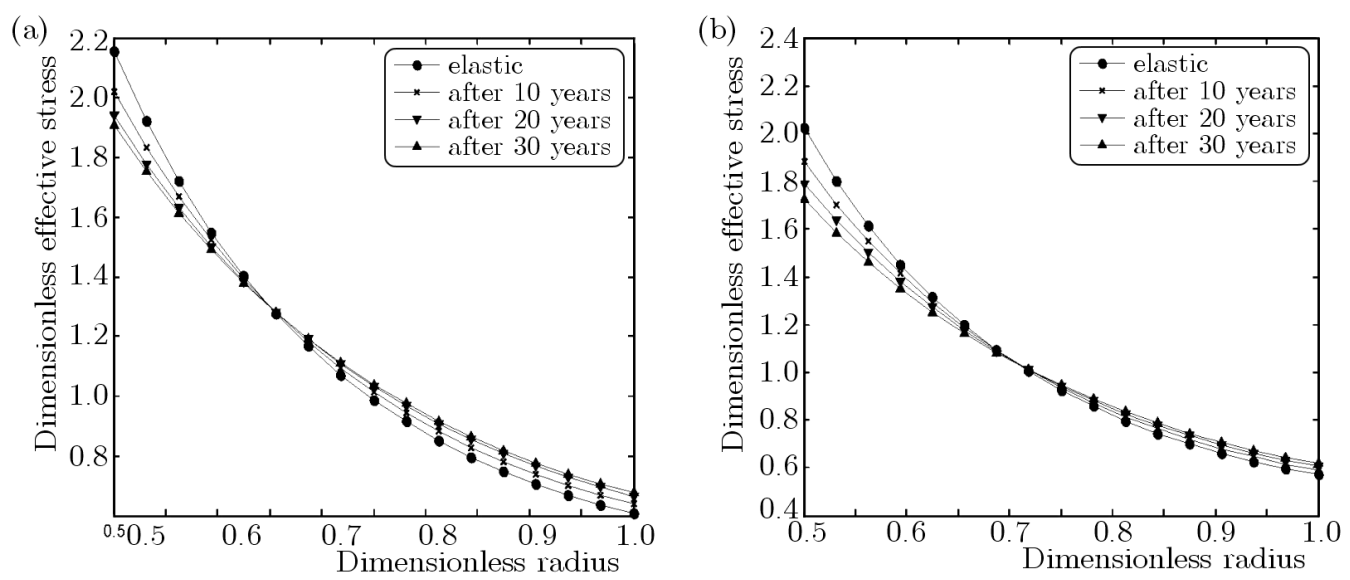

Fig. 6. Effective stress of the nano-composite cylinder (a) without and (b) with the effect of magnetic field $(H=1 \mathrm{E}+8)$

Histories of the radial strain are shown in Figs. 7a and 7b. The radial strains are compressive because of highly tensile circumferential stresses.

Histories of the circumferential strain are shown in Figs. 8a and 8b. The circumferential strains are positive due to highly tensile circumferential stresses.

Histories of the radial creep strain are shown in Figs. 9a and 9b. It is clear that the radial creep strains at zero time are zero, however, their absolute values are increasing with time due to creep. The creep strains are negative because of highly tensile circumferential stresses. 

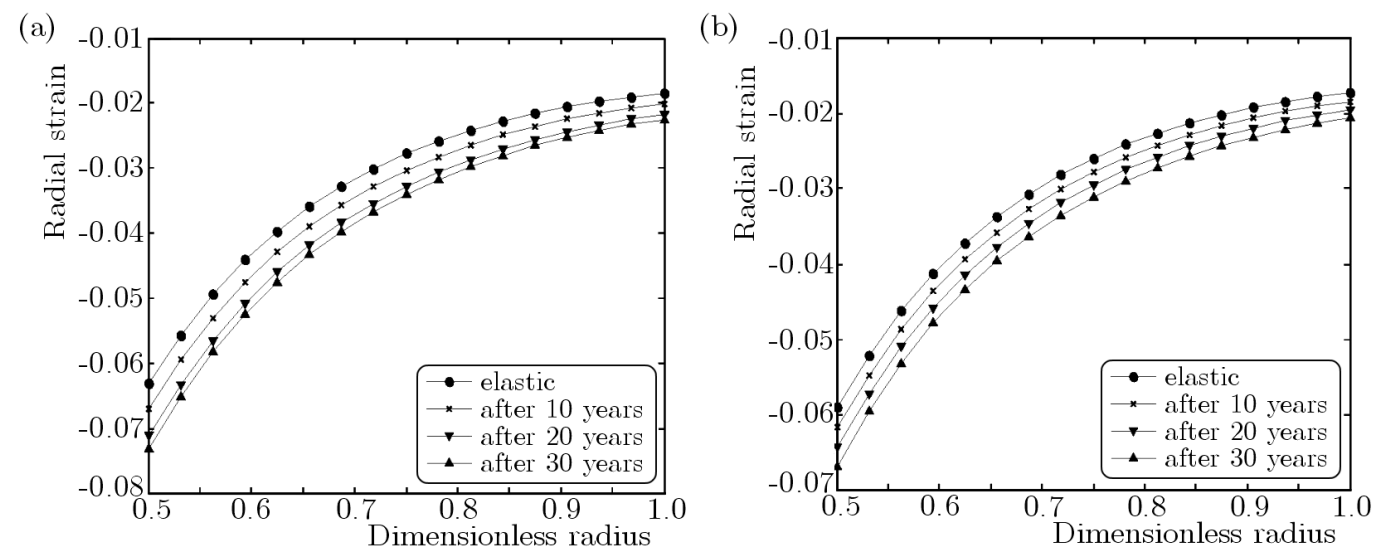

Fig. 7. Radial strain of the nano-composite cylinder (a) without and (b) with the effect of magnetic field $(H=1 \mathrm{E}+8)$
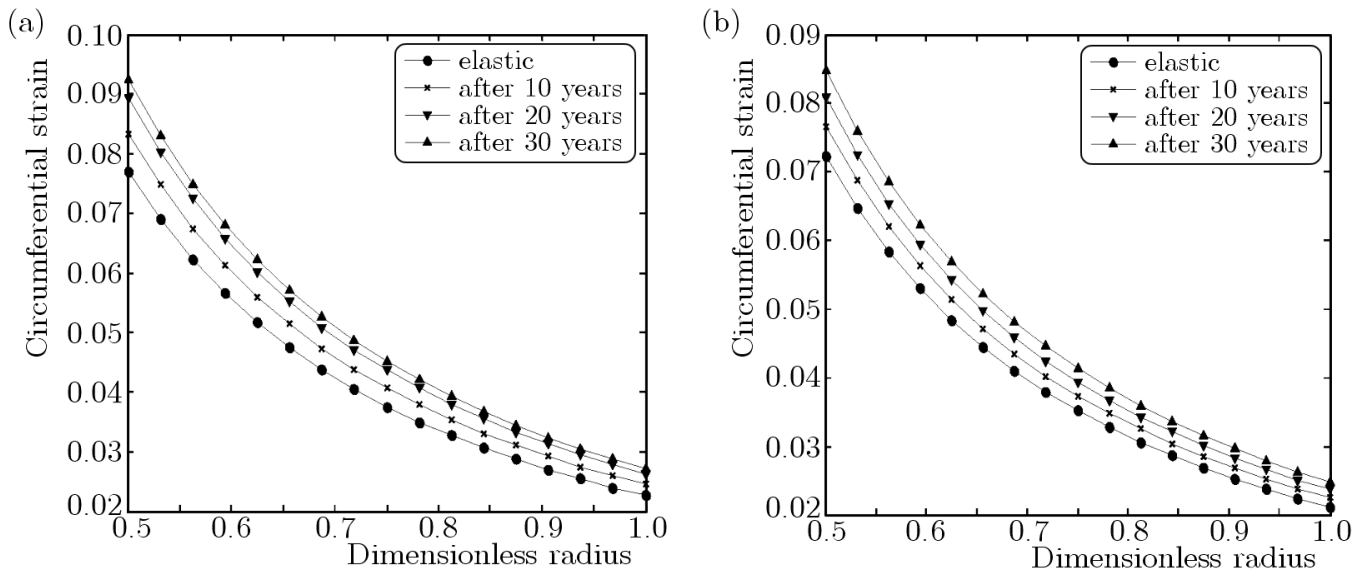

Fig. 8. Circumferential strain of the nano-composite cylinder (a) without and (b) with the effect of magnetic field $(H=1 \mathrm{E}+8)$
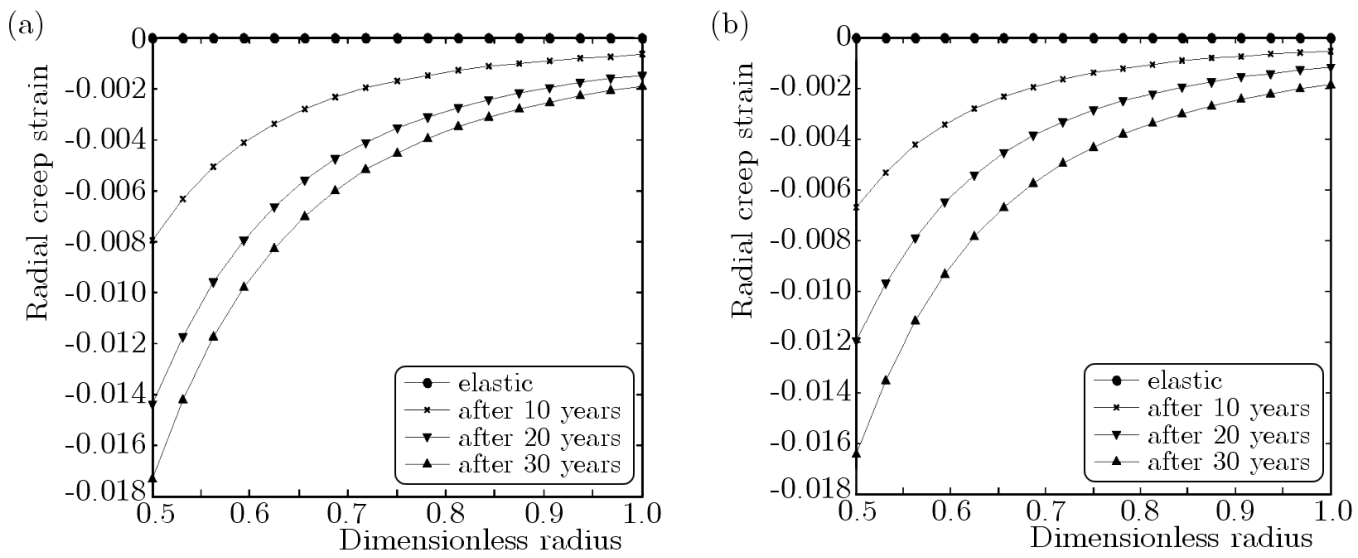

Fig. 9. Radial creep strain of the nano-composite cylinder (a) without and (b) with the effect of magnetic field $(H=1 \mathrm{E}+8)$

Histories of the circumferential creep strain are shown in Figs. 10a and 10b. Due to the incompressibility condition of the material, the circumferential strains are positive. They are also increasing with time due to creep deformation.

Generally, the stresses, strains and displacements are changing with time at a decreasing rate during life of the cylinder so that they finally approach the steady state condition. However, in the presence of a magnetic field they are all lower in magnitude. 

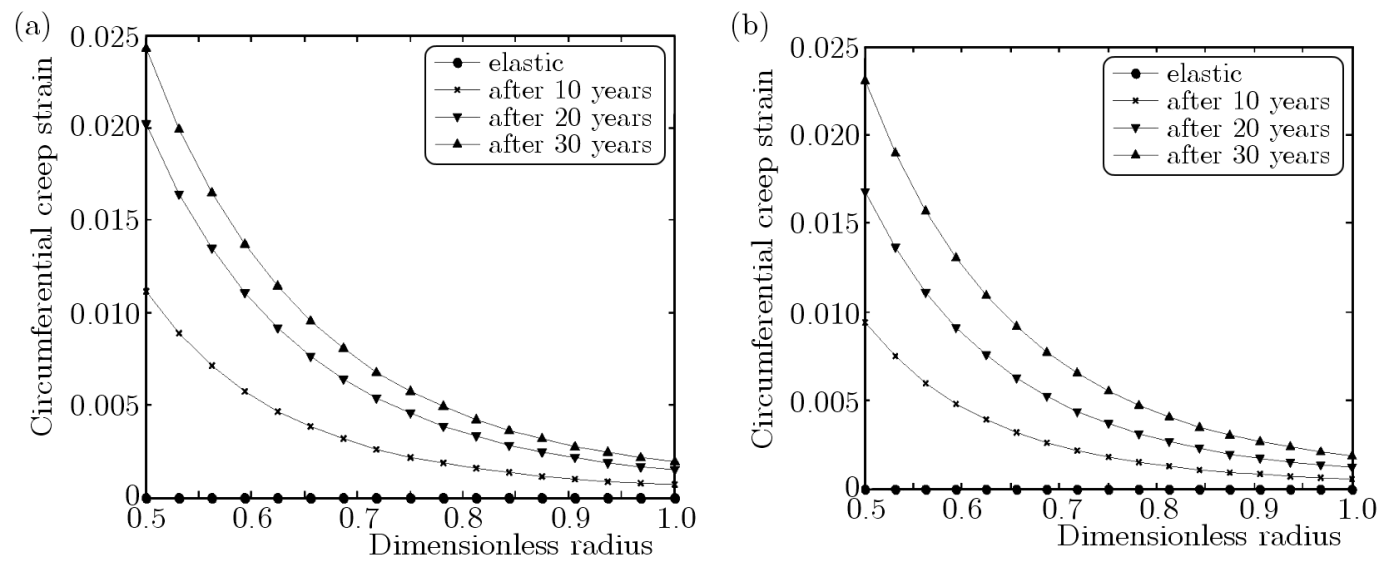

Fig. 10. Circumferential creep strain of the nano-composite cylinder (a) without and (b) with the effect of magnetic field $(H=1 \mathrm{E}+8)$

\section{Conclusion}

Time-dependent creep stress, strain and displacement analysis of a rotating thick-walled nano-composite cylinder made of polypropylene reinforced by multi-walled carbon nanotubes (MWCNTs)subjected to magnetic, thermal and mechanical load is investigated using Burgers viscoelastic creep model. The results are presented with and without the effect of magnetic field. It has been found that the radial displacement, tangential strain and absolute values of the radial strain are increasing with time at a decreasing rate so that they finally approach the steady state condition. The effective stresses are decreasing at the inner surface and increasing at the outer surface of the cylinder and approach their steady state condition after 30 years. In the presence of magnetic field stresses, the strains and radial displacement are lower in magnitude.

\section{Acknowledgement}

The authors are grateful to University of Kashan for supporting this work by giving research grant.

\section{References}

1. Barai P., Weng G.J., 2011, A theory of plasticity for carbon nanotube reinforced composites, International Journal of Plasticity, 27, 539-559

2. Bhatnagar N.S., KulkarniP.S., Arya V.K., 1984, Creep analysis of an internally pressurized orthotropic rotating cylinder, Nuclear Engineering and Design, 83, 379-388

3. Bhatnagar N.S., Kulkarni P.S., Arya V.K., 1986, Analysis of an orthotropic thick-walled cylinder underprimary creep conditions, International Journal of Pressure Vessels and Piping, 23, $165-185$

4. Ghorbanpour Arani A., Loghman A., Shajari A.R., Amir S., 2010, Semi-analytical solution of magneto-thermo-elastic stresses for functionally graded variable thickness rotating disks, Journal of Mechanical Science and Technology, 24, 2107-2117

5. Hosseini Kordkheili S.A., Naghdabadi R., 2007, Thermo-elastic analysis of a functionally graded rotating disk, Composite Structures, 79, 508-516

6. Jia Y., Peng K., Gong X.L., Zhang Z., 2011, Creep and recovery of polypropylene/carbon nanotube composites, International Journal of Plasticity, 27, 1239-1251

7. Loghman A., Ghorbanpour Arani A., Amir S., Vajedi A., 2010, Magneto-thermo-elastic creep analysis of functionally graded cylinders, International Journal of Pressure Vessels and Piping, 87, 389-395 
8. Loghman A., Ghorbanpour Arani A.,Shajari A.R., And Amir S., 2011, Time-dependent thermo-elastic creep analysis of rotating disk made of Al-SiC composite, Archive of Applied Mechanics, 81, 1853-1864

9. Mendelson A., 1968, Plasticity Theory and Applications, The Macmillan Company, New York

10. Moal P.L., Perreux D., 1994, Evaluation of creep compliances of unidirectional fiber-reinforced composites, Composites Science and Technology, 51, 469-477

11. Ohno N., Ando T., Miyake T., Biwa S., 2002, A variational method for unidirectional fiberreinforced composites with matrix creep, International Journal of Solid and Structures, 39, 159-174

12. Singh S.B., RAY S., 2002, Modeling the anisotropy and creep in orthotropic aluminum-silicon carbide composite rotating disc, Mechanics of Materials, 34, 363-372

13. Yang J.L., Zhang Z., Schlarb A.K., Friedrich K., 2006, On the characterization of tensile creep resistance of polyamide 66, Polymer, 47, 6745-6758

14. You L.H., Ou Z.Y., Zheng H., 2007, Creep deformations and stresses in thick-walled cylindrical vessels, Composite Structures, 78, 285-291

Manuscript received November 17, 2014; accepted for print August 13, 2015 